\title{
Evaluación ecocardiográfica de la función del ventrículo derecho con doppler tisular luego de una anestesia espinal
}

\author{
Echocardiographic evaluation of right ventricle function \\ with tissue doppler after spinal anesthesia
}

María Carolina Cabrera Schulmeyer ${ }^{1}$, Cristopher Araya ${ }^{2}$

\begin{abstract}
The function of the right ventricle (RV), for years forgotten, is an important predictor of perioperative morbidity and mortality, so its evaluation is fundamental. The irregular morphology of it makes difficult the use of geometric echocardiographic models classically used with the Left Ventricle (LV). Echocardiographic indices have been studied to diagnose RV dysfunction in noncardiac surgery, such as tricuspid annular systolic excursion (TAPSE), with good correlation with Magnetic Nuclear Resonance (MRI). Lately, the s' wave measurement of the RV has been validated by Tissue Doppler (TDI) to objectify its functionality. A value $\mathrm{s}^{\prime}$ greater than $9.5 \mathrm{~cm} / \mathrm{sec}$ is defined as normal, having a good correlation with the clinic and TAPSE measured by the same operator. This index has advantages such as being less operator dependent, faster to obtain and with good reproducibility, however, there are no studies that evaluate the effectiveness of this parameter independently. Objectives: To evaluate the diagnostic capacity of the $s^{\prime}$ wave in a clinical situation that is known to cause RV dysfunction, such as Spinal Anesthesia (SA). Materials and Methods: A prospective study in which ASA I patients older than 18 years were included, assigned to non-cardiac elective surgery with SA. Baseline measurements of heart rate (HR), mean arterial pressure (MAP) and $s^{\prime}$ wave were made prior SA. After the intrathecal administration of $0.75 \%$ Bupivacaine and Fentanyl, a control of the same variables was performed. For the measurement of wave $\mathrm{s}^{\prime}$ $(\mathrm{cm} / \mathrm{sec})$ a transthoracic cardiac probe ( 1 to $5 \mathrm{MHz}$ ) was used in apical window 4 cameras at the level of the tricuspid ring. Results: 57 patients were recruited (44\% women and $56 \%$ men). In all patients, there was a decrease in both MAP $(85 \pm 9 \mathrm{mmHg}$ to $74 \pm 7 \mathrm{mmHg})(p<0.01)$ and HR (from $78 \pm 12$ beats per
\end{abstract}

\section{Key words:}

Echocardiography, tissue doppler, TAPSE

Anestesióloga Universidad de Valparaíso, sede Hospital Clínico Fuerza Aérea de Chile. Santiago, Chile.

2 Residente Anestesiología y Reanimación, Universidad de Valparaíso, Chile.

Fecha de recepción: 22 de noviembre de 2018

Fecha de aceptación: 25 de julio de 2019

\section{ORCID}

https://orcid.org/0000-0002-5544-8000

Correspondencia:

Email: maría.cabrera@uv.cl 
minute $[\mathrm{bpm}]$ to $61 \pm 9 \mathrm{bpm})(\mathrm{p}<0.01)$. The decrease in the preload associated with the SA was reflected in a reduction in the wave speed $s^{\prime}$ from $11.5+5.8$ $\mathrm{cm} / \mathrm{sec}$ before SA to $9.8+5.1 \mathrm{~cm} / \mathrm{sec}(p<0.01)$. Conclusions: For years the intraoperative evaluation of the LV has been given greater importance, however, the function of the RV can also be important to consider since this is where the LV preload will be generated. The present work demonstrates that the SA, causes a compromise of RV function evidenced as a descent of the s' wave.

\section{RESUMEN}

Introducción: La función del ventrículo derecho (VD), durante años olvidada, es un importante predictor de morbimortalidad perioperatoria, por lo que su evaluación es fundamental. La morfología irregular de este dificulta el uso de modelos geométricos ecocardiográficos clásicamente utilizados con ventrículo Izquierdo (VI). Se han estudiado índices ecocardiográficos que permitan diagnosticar disfunción del VD en cirugía no cardíaca, como la excursión sistólica del anillo tricuspídeo (TAPSE), con buena correlación con resonancia nuclear magnética (RNM). Últimamente se ha validado la medición de onda s' del VD mediante doppler tisular (TDI) para objetivar su funcionalidad. Se define un valor s' mayor a 9,5 cm/seg como normal, teniendo una buena correlación con la clínica y TAPSE medido por el mismo operador. Este índice cuenta con ventajas como ser menos operador dependiente, más rápido de obtener y con buena reproducibilidad, sin embargo, no existen estudios que evalúen la eficacia de este parámetro en forma independiente. Objetivos: Evaluar la capacidad diagnóstica de la onda $s^{\prime}$ en una situación clínica que conocidamente causa disfunción de VD, como lo es la anestesia espinal (AE). Materiales y Métodos: Estudio prospectivo, en el cual se incluyeron pacientes ASA I mayores de 18 años, asignados a cirugía electiva no cardíaca con AE. Se realizó medición basal de frecuencia cardíaca (FC), presión arterial media (PAM) y onda S' previo a AE. Tras la administración intratecal de bupivacaína 0,75\% y fentanilo se realizó un control de las mismas variables. Para la medición de onda s' $(\mathrm{cm} / \mathrm{seg})$ se utilizó una sonda transtorácica cardíaca (1 a $5 \mathrm{MHz}$ ) en ventana apical 4 cámaras a nivel del anillo tricuspídeo. Resultados: Se reclutaron 57 pacientes (44\% mujeres y $56 \%$ hombres). En todos los pacientes ocurrió un descenso tanto de la PAM $(85 \pm 9 \mathrm{mmHg}$ a $74 \pm 7 \mathrm{mmHg})(p<0,01)$ como de la FC (desde $78 \pm 12$ latidos por minutos [lpm] a $61 \pm 9$ lpm) $(p<0,01)$. El descenso de la precarga asociado a la $A E$ se vio reflejado en una reducción de la velocidad de onda $\mathrm{s}^{\prime}$ desde $11,5+5,8 \mathrm{~cm} / \mathrm{seg}$ previo a AE a 9,8+5,1 cm/seg $(p<0,01)$. Conclusiones: Durante años se le ha dado mayor importancia a la evaluación intraoperatoria del VI, sin embargo, la función del VD también puede ser importante de considerar ya que es aquí donde se generará la precarga del VI. El presente trabajo demuestra que la $A E$, causa compromiso de la función del VD evidenciado como un descenso de la onda s'.

\section{Palabras clave:}

Ventrículo derecho, ecocardiografía, doppler tisular, TAPSE

\section{Introducción}

a edad de la población presenta una constante alza debida a cambios en el estilo de vida, la mejoría en los procedimientos diagnósticos y te- rapéuticos, lo que ha significado un envejecimiento sostenido de esta. Esto genera que una mayor cantidad de pacientes portadores de diferentes patologías cardiovasculares crónicas, deban someterse ya sea a cirugía cardíaca o no cardíaca. 
En el perioperatorio, durante años se le ha dado mayor importancia a la evaluación de la funcionalidad del ventrículo izquierdo (VI), sin embargo, se ha demostrado que una de las primeras funciones del miocardio que se ven comprometidas con la edad y enfermedades cardiovasculares, son la diástole (insuficiencia cardíaca con fracción de eyección preservada) y función del ventrículo derecho (VD), llegando incluso esta última a complicar la evolución de la primera[1].

EI VD al actuar como reservorio transitorio de volumen cumple un rol hemodinámico fundamental, pues es un importante determinante de la precarga del VI y, por lo tanto, del gasto cardíaco sistémico (GC). Es tanta la importancia fisiológica del VD que actualmente es sabido que, su disfunción en el perioperatorio es un factor de riesgo independiente de morbimortalidad[2], por lo que es fundamental objetivar su funcionalidad.

La información científica acerca del estudio del VD es escasa y como se mencionó anteriormente, se ha centrado exclusivamente en el VI, a tal punto que el VD ha sido denominado en la literatura científica como "la cámara olvidada"[3]. Dado el valor pronóstico que implica el VD en el perioperatorio, durante los últimos años ha surgido mayor interés en encontrar herramientas clínicas no invasivas que permitan objetivar su funcionalidad. Se han empleado diferentes técnicas para estos fines, como la angiografía convencional, angiografía con radionúclidos, la ecocardiografía y la resonancia nuclear magnética (RNM), siendo esta última considerada actualmente como la técnica "Gold estándar" para evaluar funcionalidad de $\mathrm{VD}[4]$.

La angiografía con radionúclidos fue uno de los métodos utilizados inicialmente para cuantificar fracción de eyección de VD (FEVD), sin embargo, requería la exposición del paciente a altas dosis de radiación, además de no estar siempre disponible y ser demasiado costosa. Actualmente, la RNM ha demostrado tener una buena correlación con la angiografía con radionúclidos, sin embargo, no es posible utilizarla en el intraoperatorio[4].

La ecocardiografía tiene muchas ventajas, entre ellas ser no invasiva (ecocardiografía transtorácica, ETT) o semiinvasiva (ecocardiografía transesofágica, ETE), relativamente fácil de realizar (sin olvidar que es operador dependiente), usar un equipo compatible con el escenario de pabellón, evaluación en tiempo real y el no utilizar radiación ni contraste, características que la convierten en la técnica más relevante en el intraoperatorio. Sin embargo, la evaluación mediante esta técnica no es fácil, dado la posición retroesternal del VD y su arquitectura compleja e irregular durante todo el ciclo cardíaco, lo cual dificulta (y prácticamente imposibilita) la aplicación de los modelos geométricos matemáticos clásicamente utilizados para valoración del VI[5],[6]. Es por ello que la medición de volúmenes ventriculares y FEVD mediante esta técnica sigue siendo un problema.

Dado lo anterior, en Anestesiología se han estudiado diversos índices ecocardiográficos que permitan diagnosticar fielmente disfunción del VD en cirugía no cardiaca. Estos índices se basan en el análisis del movimiento del anillo tricuspídeo con el uso de la técnica doppler, como forma de medir el acortamiento de las fibras longitudinales del VD y así objetivar su función sistólica[7]. El TAPSE (de su sigla en inglés tricuspid annular plane systolic excursión) corresponde a unos de los índices validados para este objetivo, y se basa en la medición (en $\mathrm{mm}$ ) en modo $\mathrm{M}$ del desplazamiento total que experimenta el anillo tricuspídeo desde la diástole a la sístole ventricular.

Un valor de TAPSE mayor a $15 \mathrm{~mm}$ se considera normal[8], pues ha demostrado tener una buena correlación con la FEVD medida por RNM y angiografía[9].

La medición de la velocidad de desplazamiento del anillo tricuspídeo en sístole (onda s') es un parámetro que se ha buscado validar para objetivar la funcionalidad del VD. Esto último debido a que tiene ventajas de gran significancia en el intraoperatorio, como el ser un examen rápido (10-15 segundos), menos operador dependiente y con buena reproducibilidad en las mediciones[10].

La medición de la onda s', se basa en la utilización del doppler tisular (TDI) a nivel del borde libre del anillo tricuspídeo en una ventana apical cuatro cámaras, ya sea mediante ETT o ETE.

La aplicación del TDI permite obtener una imagen típica (Figura 2) con las ondas s' (flujo intramiocárdico sistólico), e' y a' (flujo intramiocárdico diastólico), siendo la primera (en $\mathrm{cm} / \mathrm{seg}$ ) de gran utilidad para valorar la funcionalidad sistólica del VD. Valores de onda s' mayores o igual a 9,5 cm/seg se consideran como normales[11].

Estudios recientes han demostrado que este parámetro tiene una buena correlación con TAPSE y, por lo tanto, con FEVD medida por angiografía. Dado lo anterior, onda s' ha sido validada actualmente como parámetro para evaluar funcionalidad de VD[11].

Los estudios hasta ahora descritos solo han evaluado la onda s' comparándola con otros parámetros, pero ninguno ha estudiado su efectividad en forma independiente. 


\section{Objetivos}

Considerando que la anestesia espinal (AE) es una situación clínica en la cual existe una disfunción conocida del VD (por reducción aguda de la precarga), los objetivos del presente trabajo son analizar la correlación entre los cambios en la onda s' y precarga de VD, y así demostrar la capacidad de este parámetro de reflejar fielmente funcionalidad de VD.

\section{Materiales y Métodos}

Estudio prospectivo donde se incluyeron en el trabajo pacientes ASA I mayores de edad asignados a cirugía electiva no cardíaca realizadas con AE.

Todos los participantes firmaron el consentimiento informado, y el estudio fue aprobado por el comité de ética de la institución.

En pabellón, previo inicio de acto anestésico, se realizó una medición basal de frecuencia cardíaca (FC), presión arterial media (PAM) y onda s'.

Para la medición de esta última $(\mathrm{cm} / \mathrm{seg})$ se utilizó un transductor de ETT General Electric 1-5 MHz para obtener una ventana apical de cuatro cámaras, posicionando el cursor de TDI a nivel del borde libre del anillo tricuspídeo.

La AE fue realizada bajo un protocolo estándar que consistía en bupivacaína 0,75\% 12 mg y fentanilo 15 ug. Transcurridos 5 minutos y una vez logrado el nivel de anestesia necesario para la cirugía, se procedió a realizar un control de las mismas variables (FC, PAM y onda s').

Los valores previos y posteriores a la AE fueron registrados y tabulados.

El tamaño muestral se calculó en base a potencia de $80 \%$ y un error alfa de 0,01\%. Esto arrojó una muestra de 20 pacientes a estudiar. Los datos fueron procesados con sistema de STATA 10.0, y para el análisis estadístico paramétrico de las variables cuantitativas se utilizó la prueba t student. Un valor $p$ menor a 0,01 se consideró como estadísticamente significativo.

\section{Resultados}

Durante el período comprendido entre julio y agosto de 2018, un total de 57 pacientes cumplieron con los criterios de inclusión para participar en el estudio. En la Tabla 1 se especifican las características demográficas de los pacientes.

La AE fue exitosa en el total de los participantes. No hubo necesidad de convertir a Anestesia General, por lo que no se excluyeron pacientes una vez iniciado el estudio.

Las principales cirugías involucradas correspondieron al área urológica (varicocelectomía, circuncisión), coloproctológica (ligadura hemorroidal, drenaje abscesos perianales) y traumatológica (artroscopia de rodilla y osteosíntesis de tobillo). Los niveles de AE logrados durante el estudio fueron T6, T7 y T8 en el $68 \%, 24 \%$ y $8 \%$ de los pacientes respectivamente.

En la Tabla 2 se detallan los promedios de las variables hemodinámicas medidas antes y después de la AE. Tras la administración de la AE se observa una reducción tanto de la PAM y FC, ambas estadísticamente significativas.

Cabe destacar, que el manejo hemodinámico se realizó según el protocolo local de la institución independiente si el segundo registro (a los 5 minutos) de las variables aún no había sido realizado. Es decir, ningún paciente fue expuesto intencionalmente a hipotensión y/o bradicardia con la finalidad de obtener las mediciones. Tras la $A E$, además se observó una reducción estadísticamente significativa de la onda s'.

\begin{tabular}{lc}
\hline \multicolumn{2}{c}{ Tabla 1. Características de los pacientes } \\
\hline Variable & $\begin{array}{c}\text { Total de pacientes } \\
\text { ( } \mathbf{n}=\mathbf{5 7})\end{array}$ \\
Edad [promedio (IQR); años] & $31(18-48)$ \\
Peso [promedio (DE); kg] & $71(8)$ \\
Talla [promedio (DE); cm] & $168(1,02)$ \\
Sexo: masculino/femenino [n (\%)] & $32 / 25(56 / 44)$ \\
\hline
\end{tabular}

IQR: rango intercuartil; DE: desviación estándar.

Tabla 2. Variables hemodinámicas previo y posterior a la anestesia espinal (AE)

\begin{tabular}{lccc}
\hline & Pre AE & Post AE & Valor $\mathbf{p}$ \\
PAM (promedio [DE]; mmHg & $85 \pm 9$ & $74 \pm 7$ & $<0,01$ \\
FC (promedio [DE]; Ipm & $78 \pm 12$ & $61 \pm 9$ & $<0,01$ \\
Onda S' (promedio [DE]; cm/segundo & $10,5 \pm 5,8$ & $9,8 \pm 5,1$ & $<0,01$ \\
\hline
\end{tabular}

PAM: presión arterial media; FC: frecuencia cardiaca; DE: desviación estándar; AE: anestesia espinal. 


\section{Discusión}

En este estudio se demostró que luego de una $A E$ se produjo una disminución de la velocidad de acortamiento del VD medido con TDI. Además, se correlacionó bien con una disminución concomitante de la excursión sistólica del plano del anillo tricuspídeo (TAPSE). La mayor ventaja del uso del TDI estarían dadas por la facilidad de su obtención y el ser menos operador dependiente.

Tras la administración de la AE se observó un descenso de la PAM de $85 \pm 9 \mathrm{mmHg}$ a $74 \pm 7 \mathrm{mmHg}$ $(p<0,01)$ y de la FC de $78 \pm 12 \mathrm{lpm}$ a $61 \pm 9 \mathrm{lpm}(\mathrm{p}$ $<0,01$ ), efecto hemodinámico esperado debido a la simpatectomía que implica esta técnica anestésica.

Adicionalmente, existió un descenso en la velocidad de la onda s' medida por ETT, desde 10,5 $\pm 5,8$ $\mathrm{cm} / \mathrm{seg}$ a $9,8 \pm 5,1 \mathrm{~cm} / \mathrm{seg}(p<0,01)$.

La tasa de sobrevida asociada con disfunción severa de VD puede ser tan baja como 25-30\%[12], constituyendo un factor de mal pronóstico. Esto ha desviado la atención de los trabajos actuales, a encontrar una herramienta clínica lo suficientemente eficaz para diagnosticar oportunamente esta situación y, por lo tanto, guiar el manejo hemodinámico adecuado.

Jaussi et al.[13], demostraron que la prevalencia de anormalidades en el corazón derecho es frecuente en población no quirúrgica. Esto enfatiza la importancia de un diagnóstico temprano para una intervención precoz, dado que el manejo de la disfunción de VD constituye un desafío[14].

Pese que la RNM y angiografía por radionúclidos son las que tienen mayor precisión en el diagnóstico prequirúrgico de disfunción de VD, su disponibilidad en el intraoperatorio es imposible.

La ecocardiografía intraoperatoria mediante el uso de índices que evalúan contracción longitudinal y no volúmenes, como TAPSE y onda s', han ingresado como alternativa para suplir las deficiencias antes descritas. Hasta el momento, ambos índices han demostrado buena correlación con la RNM.

En el presente estudio se demostró la eficacia de la onda s' de manera independiente, es decir, no comparándola con otro método, sino que determinando su capacidad diagnóstica en una situación conocida de disfunción de VD, como lo es la AE.
Si bien no existe una caída en la velocidad peak de la onda $s^{\prime}$ bajo los valores propuestos por las diferentes guías de ecocardiografía $(<9,5 \mathrm{~cm} / \mathrm{seg})$ para diagnosticar disfunción de VD, si existe una caída estadísticamente significativa en relación directa con un descenso en la precarga del VD.

De lo anterior se puede inferir que conforme existe una reducción en el tono simpático, ocurre una caída en el retorno venoso del VD con el compromiso hemodinámico secundario a esta disfunción ventricular, lo cual se ve reflejado en una reducción estadísticamente significativa en la velocidad de la onda s'. Esto último es de mucha importancia en el intraoperatorio, pues corresponde a una herramienta diagnóstica con muchas ventajas como se mencionó anteriormente.

Además, el contar con esta herramienta nos permite buscar precoz y dirigidamente disfunción de VD cuando nos enfrentamos ante situaciones patológicas que sabemos que pueden causarla. Como por ejemplo, cuadros sépticos, embolia pulmonar, infarto del $V D$, entre otros.

Las causas de falla ventricular derecha intraoperatoria son múltiples y estas incluyen edad, mala protección miocárdica o una circulación extracorpórea muy prolongada en el caso de cirugía cardíaca[14]. La presencia de isquemia miocárdica, embolia pulmonar, uso de protamina y sobrecarga de presión y volumen de cavidades derechas son situaciones que pueden ocurrir tanto en cirugía cardíaca como no cardíaca, pudiendo complicarse la evolución de ambas.

El uso de la ETT resulta fundamental no solo para el diagnóstico de disfunción de VD, sino que también para guiar el manejo adecuado, y así mejorar considerablemente el pronóstico de nuestros pacientes.

Una de las limitaciones del estudio, es que no se realiza un análisis estadístico que permita excluir factores externos que puedan influir en la velocidad de la onda s', como por ejemplo, uso de vasopresores para manejo de hipotensión (fenilefrina, efedrina), posición del paciente (supino, litotomía), cantidad y tipo de fluidos aportados.

En el presente estudio, el objetivo sólo fue evaluar la capacidad diagnóstica de la onda s' y no su capacidad de guiar terapia hemodinámica, por lo que este estudio no permitió determinar si este parámetro tiene impacto clínico significativo, por lo que este tema será motivo de futuras investigaciones del grupo. 


\section{Referencias}

1. Obokata M, Reddy YN, Melenovsky V, Pislaru S, Borlaug BA. Deterioration in right ventricular structure and function over time in patients with heart failure and preserved ejection fraction. Eur Heart J. 2019 Feb;40(8):689-97. https://doi.org/10.1093/eurheartj/ehy809 PMID:30544228

2. Apostolakis S, Konstantinides S. The right ventricle in health and disease: insights into physiology, pathophysiology and diagnostic management. Cardiology. 2012;121(4):263-73. https:// doi.org/10.1159/000338705 PMID:22626882

3. Kossaify A. Echocardiographic Assessment of the Right Ventricle, from the Conventional Approach to Speckle Tracking and Three-Dimensional Imaging, and Insights into the "Right Way" to Explore the Forgotten Chamber. Clin Med Insights Cardiol. 2015 Jul;9:65-75. https:// doi.org/10.4137/CMC.S27462 PMID:26244034

4. Dhawan I, Makhija N, Choudhury M, Choudhury A. Modified Tricuspid Annular Plane Systolic Excursion for Assessment of Right Ventricular Systolic Function. J Cardiovasc Imaging. 2019 Jan;27(1):24-33. https:// doi.org/10.4250/jcvi.2019.27.e8 PMID:30701713

5. Napp LC, Luesebrink U, Vogel-Claussen J, Bauersachs J, Roentgen P. Two's company: double-chambered right ventricle[corrected] [corrected]. Circulation. 2013 Mar;127(9):e469-70. https:// doi.org/10.1161/CIRCULATIONAHA.112.154054 PMID:23459578

6. Rudski LG, Lai WW, Afilalo
J, Hua L, Handschumacher MD, Chandrasekaran K, et al. Guidelines for the echocardiographic assessment of the right heart in adults: a report from the American Society of Echocardiography endorsed by the European Association of Echocardiography, a registered branch of the European Society of Cardiology, and the Canadian Society of Echocardiography. J Am Soc Echocardiogr. 2010 Jul;23(7):685-713. https://doi. org/10.1016/j.echo.2010.05.010 PMID:20620859

7. Schneider M, Binder T. Echocardiographic evaluation of the right heart. Wien Klin Wochenschr. 2018 Jul;130(1314):413-20. https://doi. org/10.1007/s00508-018-13303 PMID:29556779

8. Markley RR, Ali A, Potfay J, Paulsen W, Jovin IS. Echocardiographic evaluation of the right heart. J Cardiovasc Ultrasound. 2016 Sep;24(3):183-90. https://doi. org/10.4250/jcu.2016.24.3.183 PMID:27721944

9. Kaul S, Tei C, Hopkins JM, Shah PM. Assessment of right ventricular function using two-dimensional echocardiography. Am Heart J. 1984 Mar;107(3):526-31. https:// doi.org/10.1016/00028703(84)90095-4 PMID:6695697

10. Meluzín J, Spinarová L, Bakala J, Toman J, Krejcí J, Hude P, et al. Pulsed Doppler tissue imaging of the velocity of tricuspid annular systolic motion; a new, rapid, and non-invasive method of evaluating right ventricular systolic function. Eur Heart J. 2001 Feb;22(4):340-8. https://doi. org/10.1053/euhj.2000.2296
PMID:11161953

11. Lang RM, Badano LP, Mor-Avi $V$, Afilalo J, Armstrong A, Ernande $L$, et al. Recommendations for cardiac chamber quantification by echocardiography in adults: an update from the American Society of Echocardiography and the European Association of Cardiovascular Imaging. J Am Soc Echocardiogr. 2015 Jan;28(1):1-39.e14. https://doi. org/10.1016/j.echo.2014.10.003 PMID:25559473

12. Kaul TK, Fields BL. Postoperative acute refractory right ventricular failure: incidence, pathogenesis, management and prognosis. Cardiovasc Surg. 2000 Jan;8(1):1-9. https://doi.org/10.1016/ S0967-2109(99)00089-7 PMID:10661697

13. Jaussi A, Crittin J, Bloch A. [Echocardiography of the right heart-unknown territory. Contribution of echocardiography and Doppler echocardiography to the study of the right heart]. Schweiz Rundsch Med Prax. 1989 Apr;78(17):494-7. PMID:2657968

14. Haddad F, Couture P, Tousignant $C$, Denault $A Y$. The right ventricle in cardiac surgery, a perioperative perspective: I. Anatomy, physiology, and assessment. Anesth Analg. 2009 Feb;108(2):407-21. https://doi.org/10.1213/ ane.0b013e31818f8623 PMID:19151264

15. Denault AY, Haddad F, Jacobsohn E, Deschamps A. Perioperative right ventricular dysfunction. Curr Opin Anaesthesiol. 2013 Feb;26(1):71-81. https://doi.org/10.1097/ ACO.0b013e32835b8be2 PMID:23235519 